\title{
Sobre el mecanismo de hidratación del $\mathrm{C}_{3} \mathrm{~S}$
}

A. GOLDSCHMIDT*

\section{R E S U M E N}

Se estudió la morfología y la composición, con Microsonda (EM) y Microscopía Electrónica de Barrido (SEM), de la pasta y los productos de evaporación de la fase líquida del $\mathrm{C}_{3} \mathrm{~S}$. Se obtuvieron resultados que confirman los de trabajos anteriores, ampliándose éstos, ya que se comprobó en forma directa que, morfológicamente y químicamente, los productos de la hidratación del $\mathrm{C}_{3} \mathrm{~S}$, y los obtenidos por evaporación lenta de la fase líquida del agua de amasado del $\mathrm{C}_{3} \mathrm{~S}$, son diferentes.

Químicamente no se encontró correlación, en estos productos de evaporación, entre el Si y el $\mathrm{Ca}$, lo que es un argumento a favor de la teoría topoquímica de la hidratación.

\section{N T R O D U C C I O N}

Como el cemento portland sei compone básicamente de una mezcla de silicato dicálcico y tricálcico, $\mathrm{C}_{2} \mathrm{~S}^{* *}$ y $\mathrm{C}_{3} \mathrm{~S}$ respectivamente, el endurecimiento de una pasta de cemento más agua está asociada a la hidratación de estos dos componentes, que son los que le confieren sus propiedades mecánicas. Numerosos trabajos se han realizado sobre el mecanismo de la hidratación, usando diferentes técnicas. Al revisar diferentes trabajos sobre $\mathrm{el}_{3} \mathrm{~S}$ se llega a la conclusión de que hay suficiente información como para dilucidar el verdadero mecanismo de la hidratación. Se estudiaron los efectos que tienen sobre la hidratación del $\mathrm{C}_{3} \mathrm{~S}$ el agregado de aditivos, como $\mathrm{CaCl}_{2}, \mathrm{C}_{2} \mathrm{H}_{5} \mathrm{OH}$, electrólitos, gluconato de calcio, $\mathrm{Na}(\mathrm{OH})$ y la influencia de la cantidad de $\mathrm{H}$, del vapor de $\mathrm{H}$, el efecto de la temperatura, así como también la identificación morfológica de los constituyentes de hidratación del cemento portland usando la técnica de microscopía de barrido, espectrometría de rayos X y microsonda. La cinética del proceso de hidratación del clínker ha sido asimismo objeto de un estudio intenso.

Los conceptos básicos sobre la hidratación del cemento portland se pueden encontrar en Brunauer y Copeland (1), así como (2) las principales propiedades del $\mathrm{CH}$ y del CHS, que son los productos de hidratación del $\mathrm{C}_{3} \mathrm{~S} / \mathrm{y}$ del $\mathrm{C}_{2} \mathrm{~S}$ y de la fase acuosa. Collepardi (3) observa que con un exceso de agua decrece la velocidad de hidratación, lo que se atribu-

\footnotetext{
* Laboratorio de Microanálisis, Departamento de Geología, Casilla 13518, Correo 21, Facultad de Ciencias Físicas y Matemáticas, Universidad de Chile, Santiago, Chile.

** $\quad$ En la química del cemento $\mathrm{C} \equiv \mathrm{CaO}, \mathrm{s} \equiv \mathrm{SiO}_{2}, \mathrm{H} \equiv \mathrm{H}_{2} \mathrm{O}, \mathrm{A} \equiv \mathrm{Al}_{2} \mathrm{O}_{3}, \overline{\mathrm{S}} \equiv \mathrm{SO}_{3}$.
} 
ye a que una disminución de la concentración iónica en la fase líquida, puesto que ios electrólitos aceleran siempre la hidratación, pero en cambio los alcoholes la ratardan. Odler y Skalny estudiaron la influencia del $\mathrm{CaCl}_{22}$ en la hidratación del $\mathrm{C}_{3} \mathrm{~S}$ y la formación de tobarmorita en función del tiempo y la concentración del aditivo (4); en camb:o Berger, Young y Lawrence, en otro trabajo (5), discuten el cambio morfológico del silicato de calcio hidratado (CHS) ante la presencia de $\mathrm{CaCl}_{2}$. Williamson (6), quien se manifiesia partidario de la teoría de Le Chatelier, usando la técnica de microscopía de barrido por su poder separador, hace un estudio morfológico de los constituyentes del cemento po:'iland hidratado. Kittl, Lyon, Montes y Croquevielle (7) estudiaron la cinética del proceso de la hidratación, encontrándose diferentes etapas en función del tiempo: la primera sería una hidratación instantánea; la segunda caracterizada por un aumento significativo del grado de hidratación; la tercera se puede considerar como resultante de un proceso de difusión. Trabajos posteriores confirman lo complejo de las etapas por las que pasa el proceso de hidratación del $\mathrm{C}_{3} \mathrm{~S}$ y del $\mathrm{C}_{2} \mathrm{~S}$.

Diamond (8) usa microscopía de barrido combinada con espectrometría de rayos $\mathrm{X}$ para identificar los constituyentes de la hidratación del cemento. Mehta (9) estudia los efectos de la cal sobre la hidratación de las pastas que contienen yeso, aluminato de calcio, o sulfoaluminato de calcio, mediante difracción de rayos X. Kalousek (10) señaló en 1974 las contradicciones existentes entre los puntos de vista de diferentes investigadores, en cuanto se refiere a la forma en que tiene lugar el mecanismo de hidratación, por vía de disolución o por vía topoquímica.

Berger y Mc Gregor (11) estudiaron los efectos de la temperatura en el crecimiento, nucleación y la morfología del $\mathrm{CH}$ durante la hidratación del $\mathrm{C}_{3} \mathrm{~S}$. Negro y Stafferi (12) aplican la microscopía de barrido al estudio del cemento mostrándose e identificándose una gran cantidad de los constituyentes hidratados.

Collepard: y Massida señalan (13) que la hidratación del $\beta-C_{2} \mathrm{~S}$ es similar en ciertas condiciones a la del $\mathrm{C}_{3} \mathrm{~S}$; el estudio se hace sólo y en presencia de $\mathrm{CaCl}_{2}$ ó $\mathrm{C}_{2} \mathrm{H}_{5} \mathrm{OH}$. Negro y Stafferi estudian la hidratación de cuatro tipos de cementos mediante microscopía de barrido, difracción de rayos $\mathrm{X}$ y microsonda mostrándose la formación micromorfológica, la estructura cristalina. Fujii y Kondo estudiaron la cinética del proceso de hidratación del $\mathrm{C}_{3} \mathrm{~S}$ (15) para temperaturas entre $10^{\prime \prime}$ y $50^{\circ} \mathrm{C}$ obteniéndose $\mathrm{CHS} \equiv \mathrm{C}_{1,68} \mathrm{SH}_{2,5}$. Nuevamente Murat y Negro (16) muestran la aplicación de la microscopía de barrido al estudio de diferentes puzolanas y cómo esta técn:ca permite poner en evidencia las texturas características de los productos de hidratación iniciales y constatar que cada puzolana tiene microcristales muy variados, pero que son característicos de cada muestra, concluyendo que la carbonización posterior no modifica la arquitectura microcristalina de las fases hidratadas, fenómeno observado anteriormente por difracción de rayos $\mathrm{X}$.

Kapranov (17) revisa diferentes trabajos relacionados con las teorías de la hidratación de! clínker y señala que en general no se tienen ideas claras acerca de en qué consiste tanto el mecanismo por vía topoquímica o por vía de solubilidad. Kapranov comunica brevemente fórmulas para describir el endurecimiento de los cementos y los divide en dos grupos según la teoría de Le Chatelier y según la teoría topoquímica. Particularmente se muestra partidario de la teoría topoquímica.

Ben-Dor (18) estudió el efecto térm:co de los aditivos tales como el $\mathrm{CaCl}_{2}, \mathrm{CdI}_{2}$ y el $\mathrm{CrCl}_{3}$ en la hidratación del silicato tricálcico. Chiocchio, Collepardi y Turriziani (19) estudian los efectos de la ausencia de ettringita y la resistencia de los cementos y concretos al ataque de los sulfatos. Traetteberg y Grattan-Bellew estudiaron (20) la hidratación del $\mathrm{C}_{3} \mathrm{~A}$ y del $\mathrm{C}_{3} \mathrm{~A}+\mathrm{C}_{2} \mathrm{HS}$ con y sin $\mathrm{CaCl}_{2}$ por SEM. Regourd y Hornain (21) (22) 
efectuaron una aplicación de la microscopía de barrido al estudio del clínker del cemento portland y demuestran, por una parte, la gran afinidad de los iones silícicos por el $\mathrm{C}_{3} \mathrm{~A}$ y, por otra parte, la intensidad y rapidez de la difusión del Si a través de la solución. Concluyen diciendo que en estos resultados pueden ser tomados como una nueva concepción en las teorías de la hidratación, apuntando que esto es un punto favorable a la disolución del $\mathrm{C}_{3} \mathrm{~S}$ de acuerdo con la teoría de Le Chatelier. Goto, Kaimon, Hosaca y Kondo (23) estudiaron la morfología y composición de la hidratación del $\mathrm{C}_{3} \mathrm{~S}$ usando una nueva técnica, en donde las muestras para microscopía de barrido y microsonda son atacadas al $1 \%$ $\mathrm{HNO}_{3}$-alcohol, alcohol glicérido (4:6 por volumen). Tadros, Skalny y Kalyoncu (24) estudiaron la hidratación inicial del $\mathrm{C}_{3} \mathrm{~S}$ y el estado de preaceleración explicando que las partículas de $\mathrm{C}_{3} \mathrm{~S}$ en el período temprano de hidratación llevan una carga positiva, seguido de una rápida hidrólisis de $\mathrm{C}_{3} \mathrm{~S}$ en donde el ion $\mathrm{Ca}^{2+}$ absorbe el silicio de la superficie de las partículas de $\mathrm{C}_{3} \mathrm{~S}$, reduciendo el período de disolución para iniciar el período de inducción. Fierens y Verhaegen (25) estudian cinco muestras de $\mathrm{C}_{3} \mathrm{~S}$ sometidas a diferentes tratamientos térmicos; la cinética de la disolución de la fase acuosa es medida para el ion calcio, encontrándose que la cinética para las cinco son diferentes.

Delmas (26) analiza la solución extraída de una pasta de cemento, por métodos de espectrometría de absorción, encontrando en ella: $\mathrm{K}_{2} \mathrm{O}, \mathrm{Na}_{2} \mathrm{O}, \mathrm{CaO}$, pero no silicio, lo que nos indicaría que la reacción de hidratación del cemento sería topoquímica, argumento que se. rá ampliado más adelante. En (27) se discute un trabajo de Kalousek en el cual trata sobre las primeras fases de la hidratación del $\mathrm{C}_{3} \mathrm{~S}$ y se concluye que inmediatamente de entrar en contacto con el agua se forma la interfase $\mathrm{C}_{3} \mathrm{SH}_{x}$, impermeable al agua por cerca de 2 a 6 horas; esto correspondería a un período latente de la hidratación de la pasta de cemento. La cinética del hidróxido de calcio y el crecimiento desde la solución (28) señalado por las curvas que se han obtenido, que son ecuaciones de primer orden, indican que el crecimiento empezaría inmediatamente después del contacto con $\mathrm{H}$, sin período de inducción, sugiriendo que el mecanismo de crecimiento del hidróxido de calcio es una difusión controlada.

Singh (29) (30) trata la influencia del gluconato de calcio y del cloruro de calcio en la hidratación de los cementos, estudiándose tres diferentes tipos de cementos portland, los cuales fueron estudiados con microcalorimetría, difracción de rayos $\mathrm{X}$ y análisis químico. Los resultados indican que el cloruro de calcio acelera, mientras que el gluconato de calcio retarda todas las fases de hidratación del cemento. La glucosa acelera la formación de ettringita mientras que retarda las fases de hidratación de los silicatos. Los gluconatos actúan como retardadores en la siguiente secuencia: gluconato de sodio $>$ gluconato de potaasio $>$ gluconato de magnesio $>$ gluconato de calcio. La influencia del $\mathrm{Na}(\mathrm{OH})$ en la hidratación del $\mathrm{C}_{3} \mathrm{~S}$ en pasta es estudiado en (31) y se investiga el calor acumulado durante intervalos de hasta 90 minutos comprobándose, en todos los casos, que la producción de calor es alta durante los primeros segundos y decrece rápidamente cuando la hidratación prosigue.

Fierens y Verhaegen estudiaron la hidratacion del silicato tricálcico (32) por vapor de agua y termoluminiscencia viéndose que, sometido a diferentes cambios térmicos, se modifican las características de los centros de hidratación y en particular la probabilidad de salida de los electrones del primer pozo de potencial; éstos son de una influencia marcada en la rapidez de la absorción química del agua sobre el silicato tricálcico, y las relaciones cuantitativas muestran que los centros excitados son un lugar de hidratación favorable.

Dron, Hornain y Petit (33) localizan por medio de análisis semicuantitativo de microsonda, y microscopía de barrido, en una lámina metálica insertada en la pasta, depósitos de silicatos de calcio hidratados, los cuales se forman por un proceso de trans-solución en las pastas de puzolanas granuladas. 
Kittl y Goldschmidt (34) estudian, en forma directa, las teorías de la hidratación del cemento, usando una técnica que consiste en analizar, por medio de análisis semicuantitativo de microsonda, microscopía de absorción y verificación de los resultados por absorción atómica los productos obtenidos por evaporación lenta del agua de amasado extraída por presión de una pasta de cemento portland, comprobándose y ampliando los resultados obtenidos en (26). Los resultados encontrados son un fuerte argumento para la teoría topoquímica de la hidratación del cemento, ya que no se verifica asociaciones $\mathrm{Si}$-Ca en los productos de evaporación lenta del agua de amasado del cemento portland, tanto a los $10 \mathrm{mi}$ nutos del amasado como a las 24 horas.

En (35) se trata sobre la solidificación del cemento portland remontándose hasta sus orígenes (Egipto y Grecia); más adelante hacen hincapié que aunque el crecimiento secundario de las fibrillas de gel de CHS es de una clara importancia en el desarrollo de la solidez de la pasta agua-cemento, su morfología detallada y su mecanismo de crecimiento han permanecido oscuros hasta ahora, aclarando más adelante que haciendo uso del microscopio electrónico de transmisión han obtenido una nueva comprensión de este problema. Las imágenes obtenidas muestran que las fibrillas no se asemejan a formas de caras regulares, tal y como se podría esperar de un crecimiento de cristales sólidos a partir de soluciones acuosas, y que las fibrillas individuales no son sólidas sino que aparecen finos tubos horadados, lo cual presenta un aspecto completamente nuevo del problema.

Reboul (36) utiliza el ultrasonido para investigar el mecanismo de la hidratación inical del $\mathrm{C}_{3} \mathrm{~S}$.

Kittl (37) hace una revisión del estado actual del mecanismo de hidratación del cemento, discutiendo las dos escuelas según Le Chatelier-Michelis y la topoquímica, en (38); efectúa un estudio sobre la morfología de los productos de hidratación haciendo una revisión de las diferentes teorías de la hidratación y su conexión con su morfología; muestra la importancia del espacio libre para que se desarrollen los productos como el $\mathrm{CH}$, que crecen a partir de una solución saturada, y termina diciendo que la tobermorita presenta en general una estructura que es irresoluble con los métodos actuales de la microscopía electrónica de barrido.

Valenti y Sabatelli (39) estudian la cinética de la hidratación durante las primeras 24 horas de reacción del $\mathrm{C}_{2} \mathrm{~S}$ y del $\mathrm{C}_{3} \mathrm{~S}$ conteniendo $\mathrm{Al}, \mathrm{Fe}, \mathrm{Mg}$, mediante difracción de ra. yos $\mathrm{X}$ y SEM.

Fujii y Kondo (40) hacen un estudio del mecanismo de hidratación sobre el $\beta-C_{2} S$, cuyo proceso de hidratación es similar al del $\mathrm{C}_{3} \mathrm{~S}$, resumiendo los resultados obten:dos, que son: un período de inducción y aceleración y luego un segundo estado de aceleración, presumi. blemente peculiar del $\beta-C_{2} S$.

Goldschmidt y Kittl (41) hacen un estudio morfológico del producto sólido de la evaporación de la fase líquida de una pasta de cemento portland comercial, obtenida por evaporación lenta, mediante microscopía óptica y electrónica de barrido; identificándose con microsonda las asociaciones $\mathrm{Ca}-\mathrm{Si}-\mathrm{Al}$ y los elementos aislados $\mathrm{Ca}, \mathrm{K}, \mathrm{Na}$, no encontrándose la asociación $\mathrm{Ca}-\mathrm{Si}$ aislada, lo que está de acuerdo con la teoría topoquímica. El método usado sirve para analizar con precisión los productos que se encuentran en solución en la fase líquida de una pasta de cemento.

El objetivo del presente trabajo es abordar las teorías de la hidratación del cemento mediante la técnica de Kittl y Goldschmidt $(34,41)$, la cual permite verificar en los productos sólidos de la fase líquida del agua de amasado del $\mathrm{C}_{3} \mathrm{~S}$ la existencia de las asociaciones 
como Si-Ca. Teniendo en cuenta el mecanismo básico de hidratación por vía de solución, que fue propuesto por Le Chatelier (42) y Michaelis (43) (44), se trata de una disolución de los materiales del clínker al contacto con el agua, sin que haya reacción química, y una precipitación de los productos hidratados a partir de una solución sobresaturada. Resultados experimentales (6) ya mencionados demuestran que los productos de hidratación se forman alrededor de los granos de clínker, y esta capa bloquearía inmediatamente el mecanismo de hidratación, lo que implica que debe haber un flujo de agua hacia la interfase clínker/material hidratado y un flujo de clínker disuelto hacia el exterior. El mecanismo topoquímico en cambio consiste en un flujo de agua a través de la capa hidratada y un crecimiento del material hidratado, a partir de la interfase clínker/material hidratado ; esto fue discutido en detalle por Kittl (37). Los productos solubles como el $\mathrm{CH}, \mathrm{KOH}$, $\mathrm{NaOH}$, pueden precipitar, a partir de una solución sobresaturada y migrar, cosa que no puede el CHS que es prácticamente insoluble.

Se estudiará entonces la fase líquida y sólida de una pasta de $\mathrm{C}_{3} \mathrm{~S}$, mediante microscopía óptica, microscopía de barrido y microsonda; los productos disueltos en la fase líquida nos mostrarán su composición y morfología, los cuales serán comparados con los encontrados en la pasta de $\mathrm{C}_{3} \mathrm{~S}$, con el fin de verificar la presencia de los silicatos de calcio hidratados $\mathrm{C}_{\mathrm{x}} \mathrm{H}_{\mathrm{y}} \mathrm{S}_{\mathrm{z}}{ }^{*}$ tanto en la fase líquida como en la pasta.

\section{PREPARACION DE LAS MUESTRAS}

Se mezcló $\mathrm{C}_{3} \mathrm{~S}$, fabricado en el laboratorio por métodos standard, con agua $(\mathrm{a} / \mathrm{C}=0,5)$ durante 3 minutos y se extrajo el agua de amasado por prensado en molde de hierro, recogiéndose la solución en una pipeta de plástico y se la guardó en un frasco del mismo material. Esta solución se filtró para eliminar partículas insolubles. La solución se depositó sobre una base de $\mathrm{Pt}$ para evaporar el $\mathrm{H}$. La pasta de $\mathrm{C}_{3} \mathrm{~S}$ se guardó por 7 días en una atmósfera saturada en agua y posteriormente metalizada con oro para ser estudiada con microsonda. Se analizaron 20 muestras de los residuos de evaporación de la solución y otras tantas muestras de pasta.

\section{RESULTADOS EXPERIMENTALES}

Se examinaron las muestras con una microsonda Cameca N. ${ }^{\circ} 46$, y el estudio morfológico se hizo con un microscopio electrónico Siemens de barrido; también se tomaron microfotografías con un microscopio óptico (MOR).

\section{Observación de la pasta por SEM y EM}

En la parte externa de la pasta de $\mathrm{C}_{3} \mathrm{~S}$ hidratada durante 7 días a temperatura ambiente $\left(20^{\circ} \mathrm{C}\right)$ (figs. 1 y 2 ) se pueden observar los granos de $\mathrm{C}_{3} \mathrm{~S}$ cubiertos de tobermorita (CHS), la cual es reconocida por sus cristales alargados y finos (figs. 3,4 y 5 ). Los cristales de $\mathrm{CH}$ se muestran claramente con su forma cúbica en micrografía en un poro de la pasta (fig. 6) ; tenemos también cristales de $\mathrm{CH}$ en forma hexagonal (fig. 7) sobre los granos del silicato tricálcico hidratado.

La xonotlita $\mathrm{C}_{6} \mathrm{~S}_{6} \mathrm{H}_{2}$ se presenta como cristales alargados pero cortos (fig. 8) en comparación con el CHS; ellos están bien formados y entrelazados al azar.

Estos son los productos más representativos de la hidratación del $\mathrm{C}_{3} \mathrm{~S}$ encontrados en la pasta.

* $\mathrm{x}, 1,0,3, \mathrm{y}=1,0,8, \mathrm{z}, 1$ 


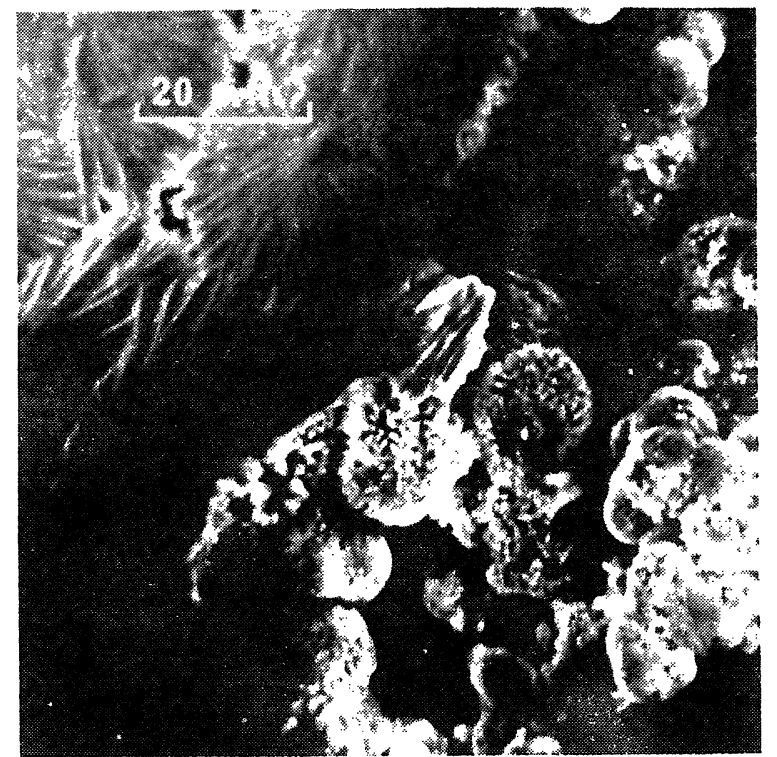

Fig. 1.-Superficie externa de una pasta de $C_{3} S$ hidratada 7 dias. Granos de $C_{3} S$ cubiertos de tobermorita (CHS) y rodeados de portlandita (CH). Microscopía Electrónica de Barrido (SEM).

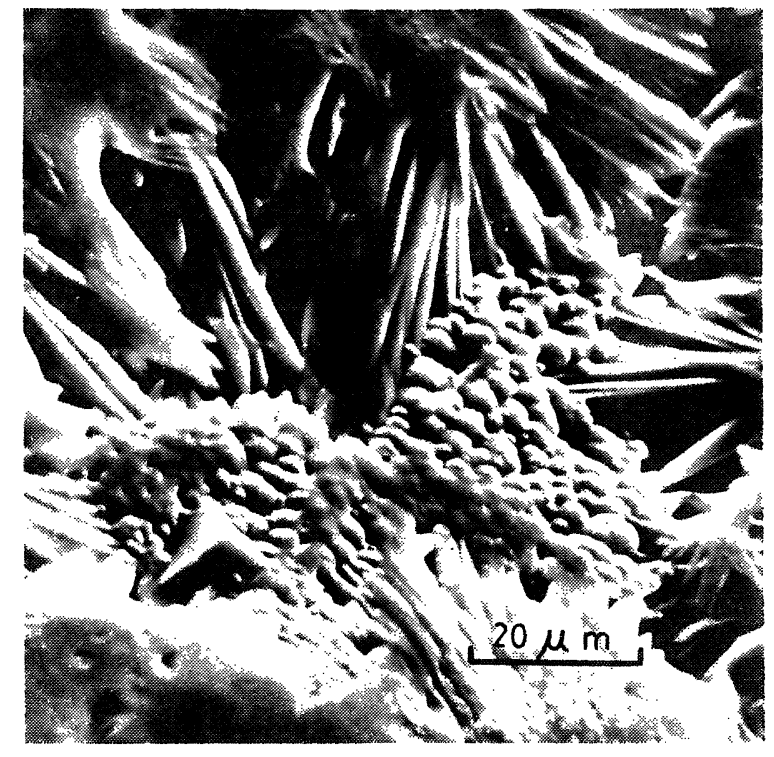

Fig. 2.-Superficie externa de la pasta anterior. Cristales de portlandia $(\mathrm{CH})$; en forma de agujas $y$ cúbicos. Microscopia Electrónica de Barrido (SEM).

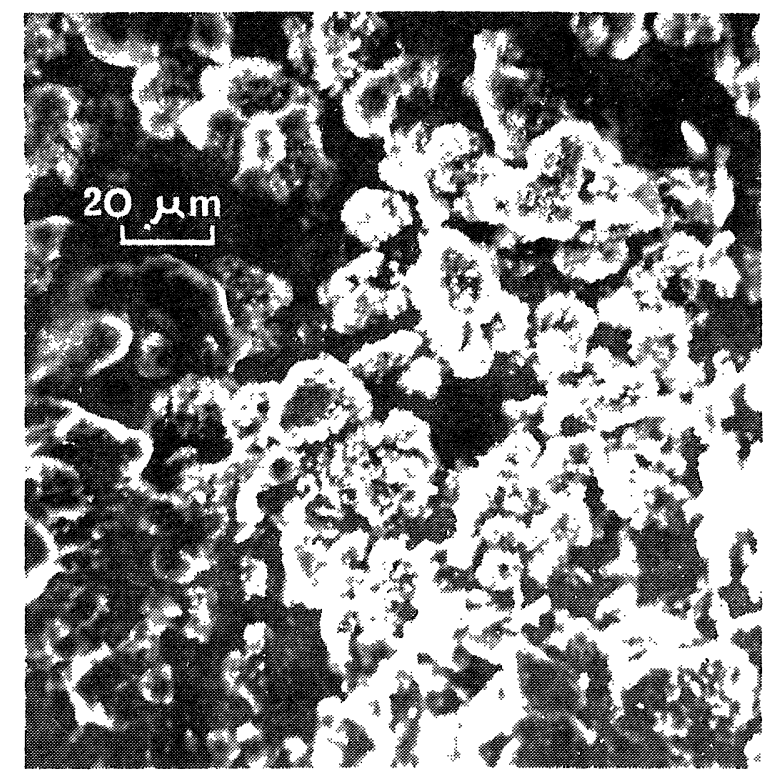

Fig. 3.-..GránuJos de $c_{3} \mathrm{~s}$ hidratados 7 dias. Mieroscopia nectrónica de Marrido (SWM).

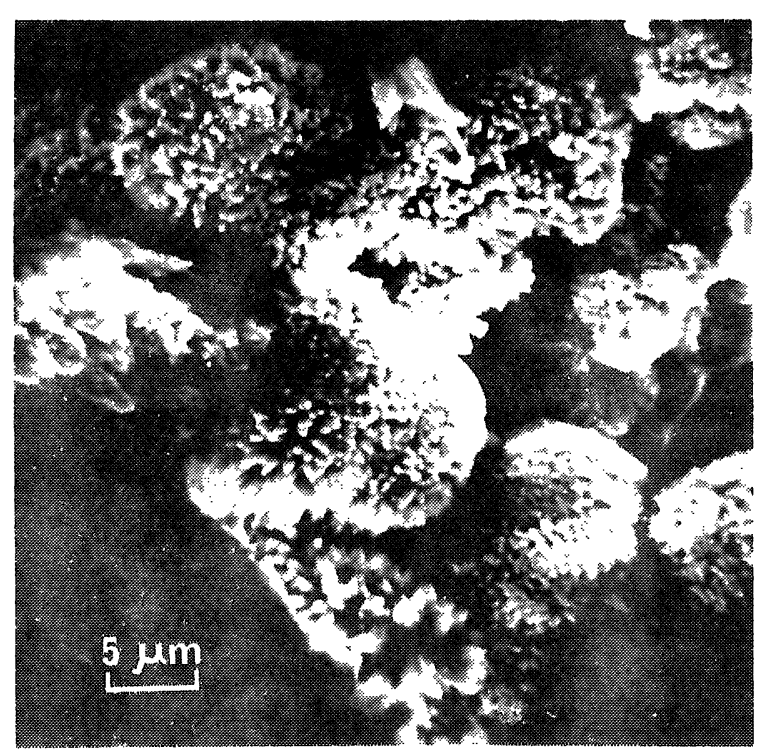

Fig. 4.-úranulos de $C_{3} s$ hidratados 7 dias. Microscopia Nectronica de Barrido (SWM). 


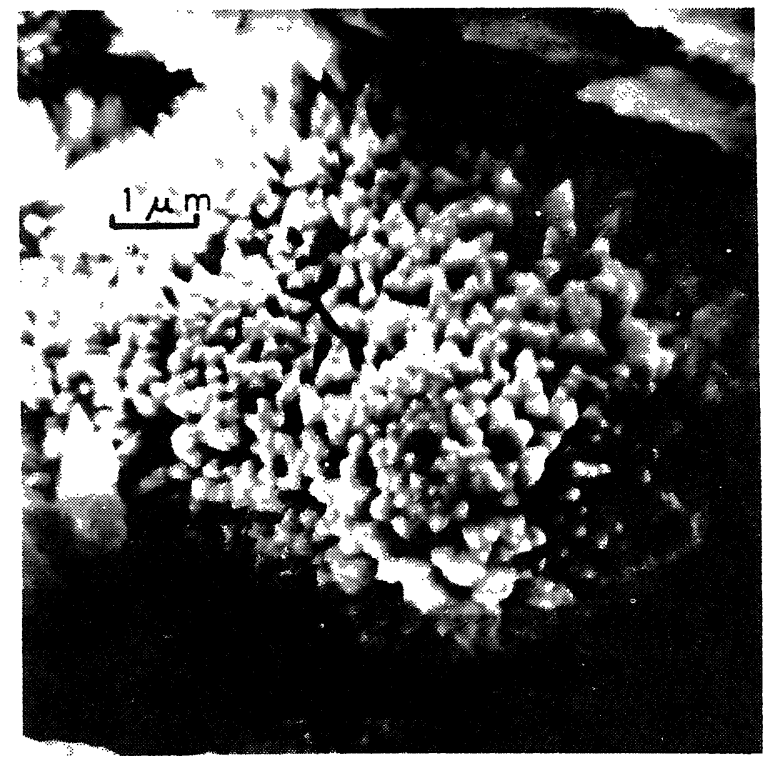

Fig. 5.- Un gránulo de $\mathrm{C}_{3} \mathrm{~S}$ de la foto anterior. Microscopía Electrónica de Barrido (SEM).

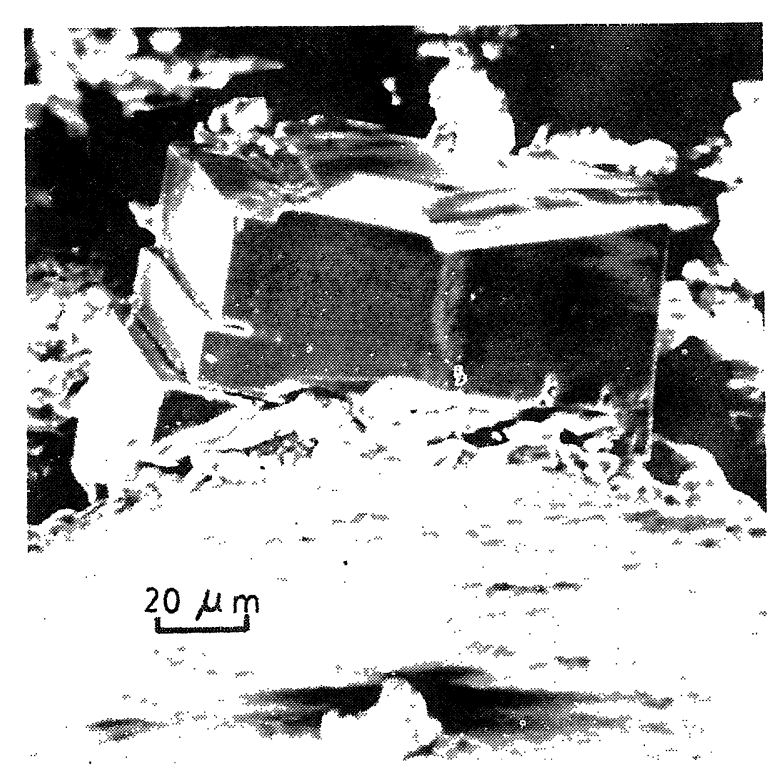

Fig. 6.-Cristales de Portlandita (CH) de formas cúbicas superpuestas, en un poro superficial de la pasta de $\mathrm{C}_{3} \mathrm{~S}$. Microscopía Electrónica de Barrido (SEM).

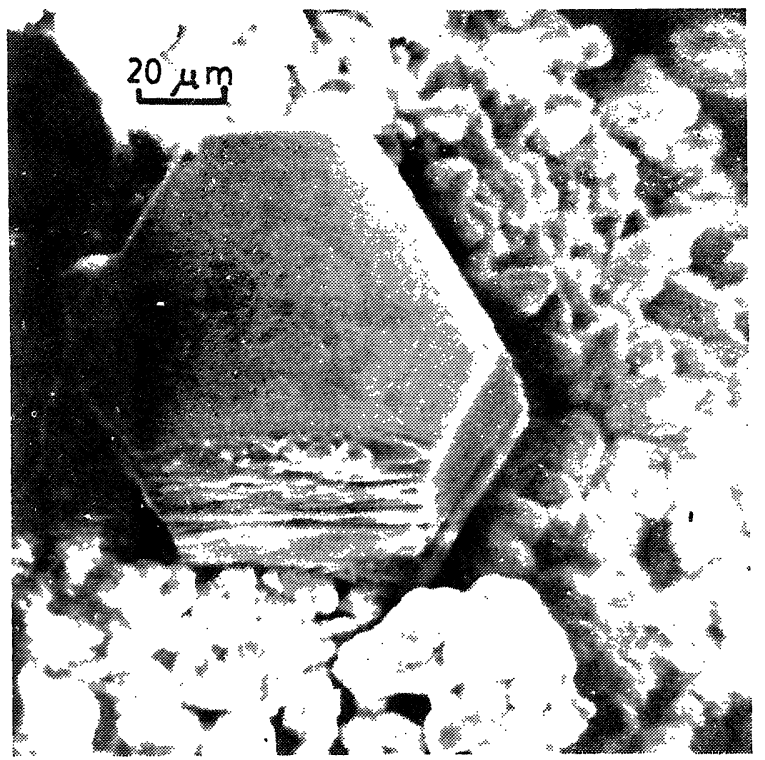

Fig. 7.-Cristal de portlandita, rodeado de granulos de $C_{3} S$ hidratados. Microscopia Electronica de Barrdo (SLM).

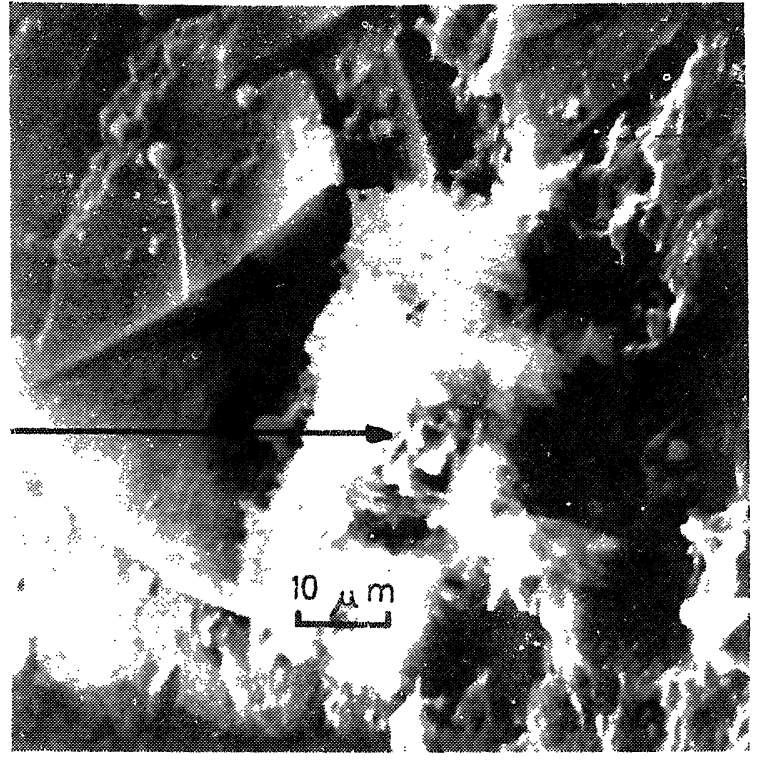

Fig. 8.-Cristales de xonotlita $\mathrm{C}_{6} \mathrm{~S}_{6} \mathrm{H}_{2}$ (señalados por la flecha), rodeatos de portlandita. Mieroscopia wectrónica de Barrido (SENI). 
Observación de los productos sólidos obtenidos por evaporación de la fase líquida del agua de amasado del $\mathrm{C}_{3} \mathrm{~S}$

Las primeras micrografías que se tomaron fueron con un microscopio óptico (MOR). La fig. 9, que es en general la más representativa de ellas, nos destaca una partícula redonda irregular rodeada de otras angulosas que fueron identificadas (EM) como CH. Esta partícula señalada con una flecha contiene en su centro agrupaciones de silicatos que se denotan por un color obscuro.

A continuación se observaron con SEM la misma partícula de la fig. 9, lo que nos demuestra con su poder separador (fig. 10) diferentes agrupaciones de iones de silicio (zonas obscuras) y que difieren claramente por su morfología del resto del soporte de portlandita (zona clara). Continuando el estudio con (EM) de la misma partícula de las figs. 9 y 10, la fig. 11 que muestra la micrografía de absorción, nos confirma en forma fehaciente el aislamiento de estos silicatos (zona obscura). La micrografía de distribución (fig. 12) nos confirma nuevamente la presencia de silicio y la falta de asociación con respecto al calcio (fig. 13) en donde el calcio rodeando al silicio no forma un compuesto con él. Podemos confirmar esto, examinando las zonas adyacentes en donde la distribución del silicio y calcio (figs. 14 y 15) se ven sin correlación alguna (EM) y el examen de dicha zona por (SEM) nos confirma este resultado (fig. 16).

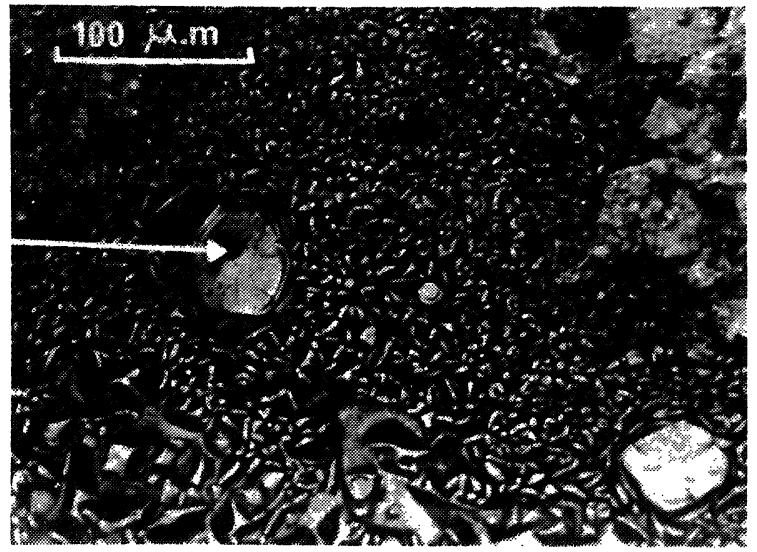

Fig. 9.-Particulas de silicio (señalado por una flecha), rodeadas de portlandita en fase sólida obtenida por la evaporación lenta de la fase líquida de la pas. ta de $\mathrm{C}_{3} \mathrm{~s}$. Microscopia Optica.

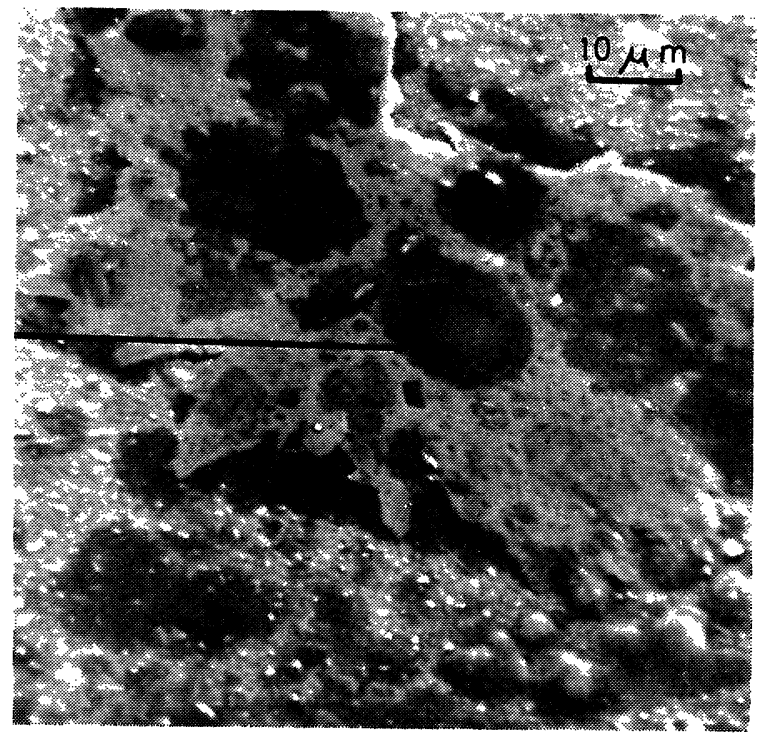

Fig. 10._Particulas de Si marcadas con una flecha en la figura anterior. Microscopía Electrónica de Barrido (SEM). Observese la morfología irregular del soporte de la portlandita. 


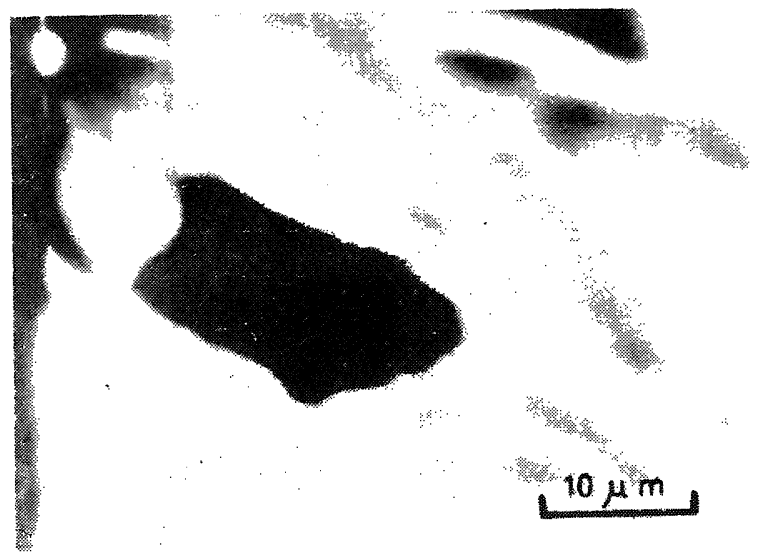

Fig. 11.-La partícula de Si de la Fig. 10. Observada por Microscopia de Absorción EM.

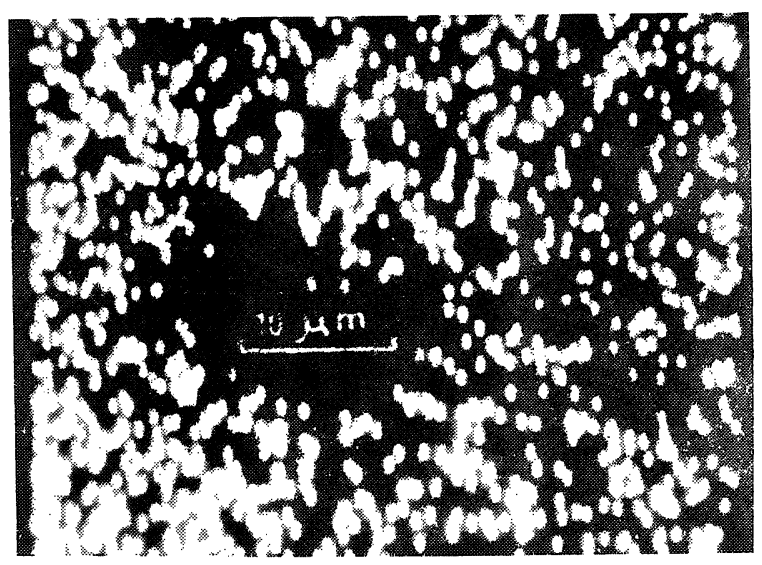

Fig. 13.-Microanálissis (EM) de la misma zona de las Figs. 10, 11, 12. Mostrando la distribución de calcio. Obsérvese la carencia de él en la partícula de Si. (Fig. 12).

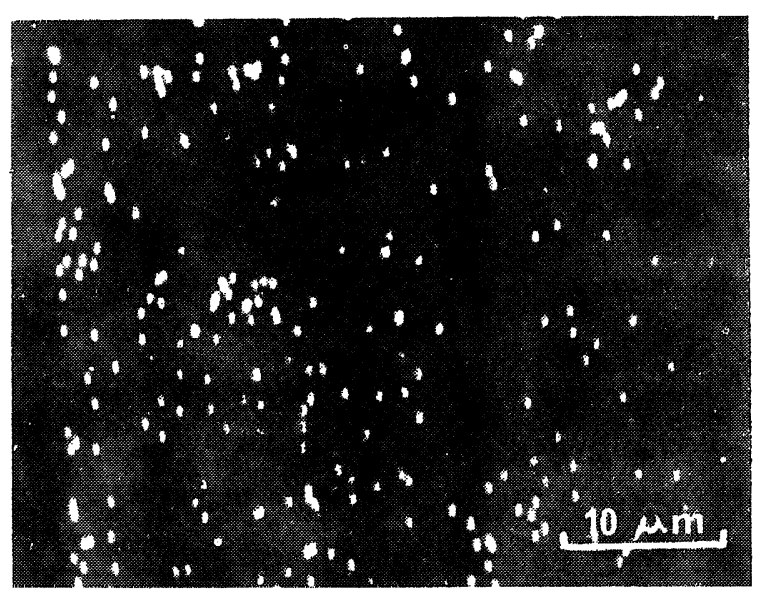

Fig. 15.-Microanálisis (EM) de la distribución de S!, en la misma zona anterior. Obsérvese que no hay correlación con la distribución de Ca (Wig. 14).

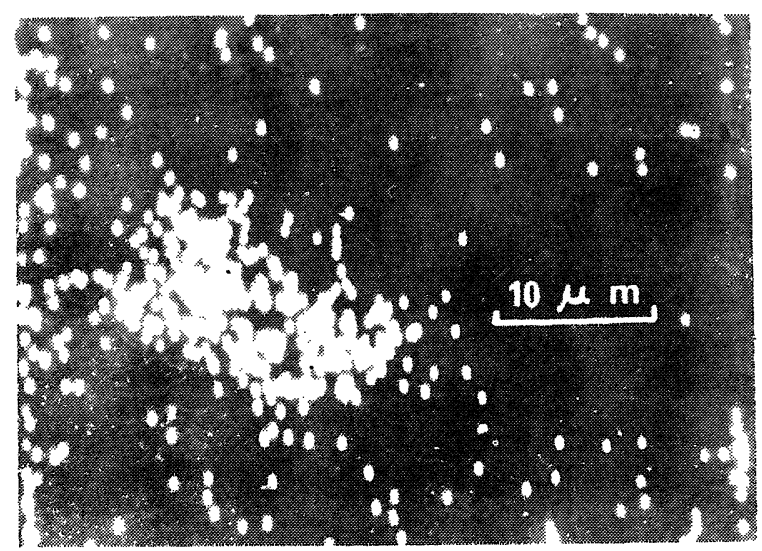

Fig. 12.-Microanálisis (EM) de la partícula de silicio de la Fig. 11. Obtenida por evaporación de la fase liquida. Obsérvese la distribución del silicio.

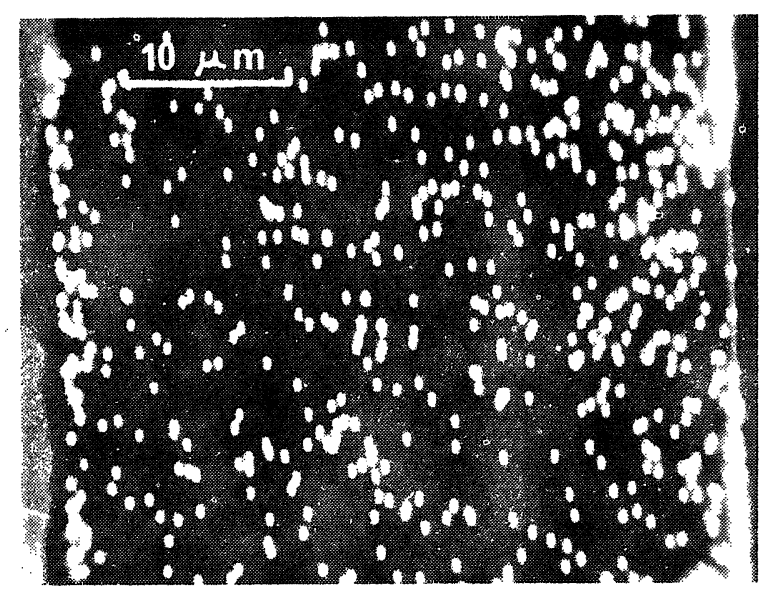

Fig. 14.-Microanálísis (EM). De una zona de la fase sóllda, obtenida por la evaporación lenta de la fase líquida. Mostrando la distribución del Ca.

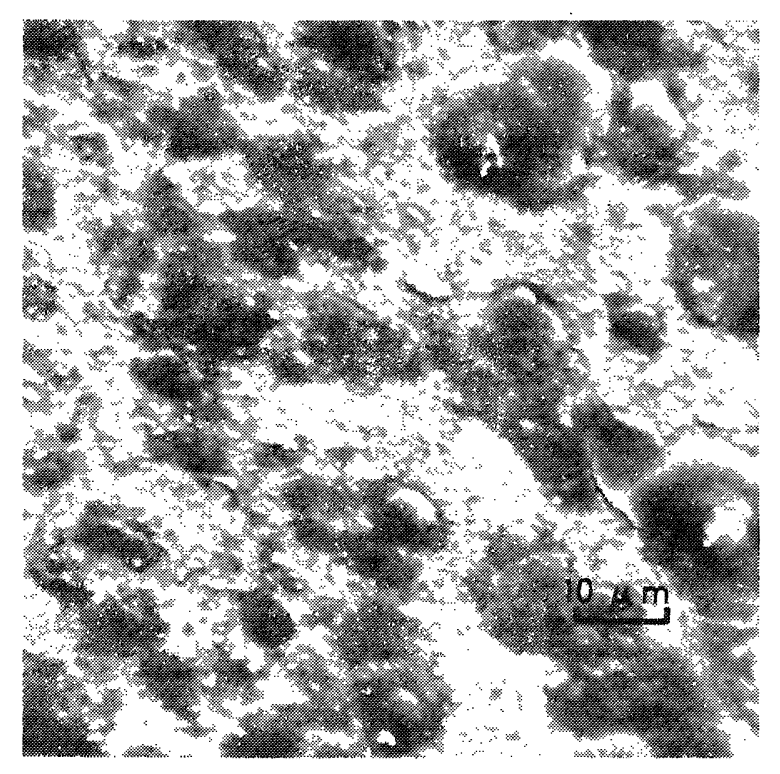

Fig. 15.-Micrografía por SEM de la misma zon: de las rigs. 14 y 15. Obsérvese la distribución al azar de las partículas, claras $\mathrm{Ca}$ y obscuras $\mathrm{Si}$. 


\section{I S C U S I O N}

Si consideramos como mecanismo topoquímico, que los minerales del clínker entran inicialmente en una reacción química sin que haya solución y se disuelven luego los productos solubles de estas reacciones, los resultados experimentales obtenidos en este trabajo nos demuestran al analizar la pasta de $\mathrm{C}_{3} \mathrm{~S}$ y los componentes sólidos de la fase líquida del agua de amasado que no hay productos de hidratación por vía solución, ya que éstos deberían disolverse al contacto con el agua para formar tobermorita.

Morfológicamente y químicamente, los productos en la pasta y el agua de amasado son diferentes, no identificándose en estos últimos ninguna asociación $\mathrm{Si}-\mathrm{Ca}$, lo que es un fuerte argumento en favor de la teoría topoquímica*, ya que estos resultados implicarían que no hay disolución de los minerales del clínker al contacto con el agua, sino más bien una reacción química sin disolución. Lo que se podría afirmar es la formación de iones difundidos a la solución, pero no así la formación de productos de hidratación insolubles dentro de la solución.

\section{A G R A D E C I M I E N T O S}

A P. Kittl por sus sugerencias durante la realización de este trabajo y la lectura del manuscrito; a J. C. Olavarría y M. Ramos, deì Laboratorio de Microscopía Electrónica de IDIEM, por su colaboración en el uso del SEM, y a M. Ramos por el trabajo fotográfico.

\section{B I B L I O G R A F I A}

(1) Brunauer, S. and Copeland, L. E.: “The Chemistry of Concrete". Scientific American, pp. 81-92, 1964

(2) Brunauer, S. and Greenberg, S. A.: "The Hydration of tricalcium Silicate and $\beta$-Dicalcium Silicate at Room Temperature”. Symposium International on the Chemistry of Cement, paper 3-1, pp. 135-163, Washington, D. C., 1960.

(3) Collepardi, M. y Massidda, L.: "Hydration of tricalcium Silicate". 73" Annual Meeting of the American Ceramic Society, pp. 419-422, Appril (1971). J. A. C. Soc., Vol. 54, N" 9, 1974.

(4) Odler, I. and Skalny, J.: "Influence of Calcium Chloride on paste hydration of tricalcium Silicate". Journal of American Ceramic Society. Vol. 54, N. 7, pp. 362-363, 1971.

(5) Berger, R. L., Young, J. F. y Lawrence, F. V.: "Discussion of Paper Morphology and Surface proprieties of Hydrated tricalcium silicate Paste by M. Collepardi", Cement and Concrete Research. Vol. 2, pp. 633-636, 1972.

(6) Williamson, R. B.: "Solidification of Portland cement". Material Science, Vol. 15, N." 3, pp. 169-285. 1972.

(7) Kittl, P., Lyon, P., Montes, S. y Croquevielle, E.: "Estudio sobre la hidratación del Clínker". Revista del IDIEM, Vol. 12, N.”2, pp. 94-102, 1973.

(8) Diamond, S.: "Identification of Hydrated Cement constituents using a Scanning Electron Microscope" Cement and Concrete Research. Vol. 2, pp. 617 632, 1972.

* Se entiende luego de eliminar lentamente, por evaporación, el H. 
(9) Menta, P. K.: "Effect of Lime on Hydration of Pastes Containing Gypsum and Calcium Aluminates or Calcium Sulfoaluminate". Journal of the American Ceramic Society, Vol. 56, N.0 6, pp. 315-319, 1973.

(10) KalouseK, L.: "Procesos de hidratación en las primeras fases del cemento". 6." Congreso Internacional de la Química del Cemento (1974), Moscú.

(11) Berger, R. L. y Mc Gregor, J. D.: "Effect of Temperature and Water-Solid Ratio on Growth of $\mathrm{Ca}(\mathrm{OH})_{2}$ Cristals Formed During Hydration of $\mathrm{Ca}_{3} \mathrm{SiO}_{5}$ ". Journal of the American Ceramic Society, Vol. 56, N.o 2, pp. 73-78, 1973.

(12) Negro, A. y Stafferi, L.: “Application du microscope électronique à Balayage à l'etude du ciment et de ses produits d'hydratation". Revue des Matériaux N.o 684, pp. 5-14, 1973.

(13) Collepardi, M. y Massida, L.: "Hydration of Bata Dicalcium Silicate Alone and in the Presence of $\mathrm{CaCl}_{2}$ or $\mathrm{C}_{2} \mathrm{H}_{5} \mathrm{OH}$ ". Journal of the American Ceramic Society. Vol. 56, N. ${ }^{\circ} 4$, pp. 181-183, 1973.

(14) Negro, A. y Stafferi, L.: “Application du Microscope électronique à l'étude du ciment et des produits d'hydratation". 2.० partie: pates et mortiers. Revue des Materiaux de Construction n. 686, pp. 17-24, 1974.

(15) FUJII, K. y Kondo, W.: "Kinetics of hydration of tricalcium silicate". Journal of the American Ceramic Society. Vol. 57, N.॰ 11, pp. 492-497, 1974.

(16) Murat, M. y Negro, A.: “Application de la microscopie électronique à balayage à l'etude des laitiers et de leurs produits d'hydratation à court terme". Matériaux et Constructions, Vol. 7, N.* 40, pp. 253263, 1974 .

(17) KaPRanov, W. W.: "El mecanismo de hidratación de los aglomerantes". Cement and Concrete Research, Tomo 5, pp. 15-24, 1975

(18) Ben-Dor, L., Dror. Perez and SARing, S.: "Thermal Study of the Effect of Additives on the Tricalcium Silicate”. Journal of the American Ceramic Society, Vol. 58, N. 34, pp. 87-89, 1975.

(19) Chrocchio, G., Collepardi, M. and Turriziani, R.: "Substituted Hydrated Calcium Silicates Obtained in Autoclave Hydration". Journal of the American Ceramic Society. Vol. 58, N.* 5-6, pp. 185-188, 1975.

(20) Traetteberg, A. y Grattan-Bellew, P. E.: "Hydration of $3 \mathrm{CaO} \mathrm{Al}_{2} \mathrm{O}_{3}$ and Gypsum with and Without $\mathrm{CaCl}_{2}$ ", Journal of the American Ceramic Society. Vol. 58, N.॰ 5-6, pp. 221-227, 1975.

(21) Regourd, M. et Hornain, H.: "Applications de la Microscopie électronique à balayage". Revue des Matériaux de Construction, N.*693, pp. 73-87, 1975.

(22) Regourd, M., Hornain, H. and Mortureux, B.: "Evidence of Calcium Silicoaluminates in Hydrated mixtures of tricalcium Silicates and Tricalcium Aluminate". Cement and Concrete Research. Vol. 6, pp. 733-740, 1976.

(23) Goto, S., KaImon, M., HosaKa, G. y Kondo, R.: "Composition and Morphology of Hydrated tricalcium Silicate". Journal of the American Ceramic Society. Vol. 59, N." 7-8, pp. 281-284, 1976.

(24) Tadros, M. E., Skalny, J. and Kalyoncu, R. S.: "Early Hydration of Tricalcium Silicate". Journal of the American Ceramic Society. Vol. 59, N.: 7-8, pp. 344-347, 1976

(25) Fierens, P. and Verhaegen, J. P.: "Hydration of tricalcium Silicate in Paste-Kinetics of Calcium Ions Dissolution in the Aqueous Phase". Cement and Concrete Research. Vol. 6, pp. 337-342, 1976. 
(26) Delmas, P.: "Evolution de la composition centésimale de la phase liquide interstitielle d'un ciment hydraté et d'un béton léger". Revue des Matériaux de Construction, N.0 702, pp. 291-295, 1976.

(27) Maycock, J. N., Skalny, J. P. and Kalyoncu, R. S.: "A Discussion to the Presentation of Dr. G. Kalousek on the Hydration Processes at the Early Stages of Cement Hardening". Comunicacion personal.

(28) Maher, E. Tadros, Skalny, J. and Kalyoncu, R.: "Kinetics of Calcium Hydroxide Crystal Growth from Solution". Journal of Colloid and Interface Science, Vol. 55, N.o 1, pp. 20-23, 1976.

(29) SingH, N. B.: "Influence of Calcium Gluconate with Calcium Choride or Glucose on the Hydration of Cements". Cement and Concrete Research, Vol. 5, pp. 545-550, 1975.

(30) Singh, N. B.: "Effect of Gluconates on the Hydration of Cement". Cement and Concrete Research. Vol. 6, pp. 455-460, 1976.

(31) SpIerings, G. S. C. M. and Stein, H. N.: "C ${ }_{3}$ A, Hydration, NaOH Solution". Cement and Concrete Research. Vol. 6, N.* 4, pp. 489-496, 1976.

(32) Fierens, P. and Verhaegen, J. P.: "Hydration of Tricalcium Silicate by Water Vapour". Cement and Concrete Research, Vol. 5. pp. 587-596, 1975.

(33) Dron, R., Hornain, H. et Petit, P.: "Localisation du silicate de calcium hydraté dans les pâtes de laitier granulé". C. R. Acd. Sc. Paris, t. 280, pp. 187-189, 1975.

(34) Kittl, P. y Goldschmidt, A.: "Sobre la constitución química del agua de amasado y las teorías de la hidratación del cemento". Materiales de Consirucción, N.o. 168, pp. 21-25, 1973.

(35) Double, D. D. y Hellawell, A.: "La solidificación del cemento". Scientific American, pp. 62-69, 1977.

(36) Reboul, J. P.: "The Hydraulic Reaction of Tricalcium Silicate observed by microwave Dielectric measurements". Revue de Physique Appliquée, Tomo 13, pp. 383-386, 1978.

(37) Krttr, P.: "Estado atual do mecanismo de hidratacao do cimento". Revista Brasileira de Technología, Vol. 8, pp. 53-62, 1977.

(38) Krttl, P. Castro, H. y Bretas, F. S.: “Morfología de los productos de hidratación del clinker". Revista del IDIEM, Vol. 16, N.’3, pp. 124-130, 1977.

(39) Valenti, G. L., Sabatelli, V. and Marchese, B.. "Hydration Kinetics of tricalcium silicate solid solutions at Early Ages". Cement and Concrete Research, Vol. 8, pp. 61-72, 1978.

(40) KInJiRo FuJII and Wakichi Kondo.: "Rate and Mechanism of Hydration of Dicalcium silicate". Journal of the American Ceramic Society, Vol. 62, N." 3-4, pp. 161-167, 1979.

(41) Goldschmidt, A. y Kitrl, P.: "Estudio de la morfología del producto de la evaporación de la fase líquida de una pasta de cemento Portland comercial". Revista del IDIEM, Vol. 18, N.' 1, pp. 34-38, 1979.

(42) Le Chatelier, H.: “Comptes Rendus” Vol. 94, pp. 715, 1883.

(43) Michaelis, W.: Chem. Seit, Vol. 3, pp. 983, 1893.

(44) Michaelis, W.: Koll. Zeit., Vol. 5, N.” 1, pp. 9, 1909. 